\title{
A randomised trial of glucocorticoids in acute-stage allergic bronchopulmonary aspergillosis complicating asthma
}

\author{
Ritesh Agarwal ${ }^{1}$, Ashutosh N. Aggarwal ${ }^{1}$, Sahajal Dhooria ${ }^{1}$, \\ Inderpaul Singh Sehgal ${ }^{1}$, Mandeep Garg ${ }^{2}$, Biman Saikia ${ }^{3}$, Digambar Behera ${ }^{1}$ \\ and Arunaloke Chakrabarti ${ }^{4}$
}

\begin{abstract}
Affiliations: ${ }^{1}$ Dept of Pulmonary Medicine, Postgraduate Institute of Medical Education and Research (PGIMER), Chandigarh, India. ${ }^{2}$ Dept of Radiodiagnosis and Imaging, Postgraduate Institute of Medical Education and Research (PGIMER), Chandigarh, India. ${ }^{3}$ Dept of Immunopathology, Postgraduate Institute of Medical Education and Research (PGIMER), Chandigarh, India. ${ }^{4}$ Dept of Medical Microbiology, Postgraduate Institute of Medical Education and Research (PGIMER), Chandigarh, India.
\end{abstract}

Correspondence: Ritesh Agarwal, Dept of Pulmonary Medicine, Postgraduate Institute of Medical Education and Research (PGIMER), Chandigarh 160012, India. E-mail: agarwal.riteshdoutlook.in

ABSTRACT Whether use of high-dose steroids in acute-stage allergic bronchopulmonary aspergillosis (ABPA) is associated with superior outcomes is not known. Herein, we compare the efficacy and safety of two glucocorticoid protocols in ABPA.

Treatment-naive ABPA subjects randomly received either high-dose or medium-dose oral prednisolone. The primary outcomes were exacerbation rates and glucocorticoid-dependent ABPA after 1 and 2 years, respectively, of treatment. The secondary end-points were composite response rates after 6 weeks, improvement in lung function, time to first exacerbation, cumulative dose and adverse effects.

92 subjects (high-dose $n=44$, medium-dose $n=48$ ) were included in the study. The numbers of subjects with exacerbation after 1 year (high-dose $40.9 \%$ versus medium-dose $50 \%, \mathrm{p}=0.59$ ) and glucocorticoiddependent ABPA after 2 years (high-dose $11.4 \%$ versus medium-dose $14.6 \%, \mathrm{p}=0.88$ ) were similar in the two groups. Although composite response rates were significantly higher in the high-dose group, improvement in lung function and time to first exacerbation were similar in the two groups. Cumulative glucocorticoid dose and side-effects were significantly higher in the high-dose group.

Medium-dose oral glucocorticoids are as effective and safer than high-dose in treatment of ABPA.

@ERSpublications

Medium-dose glucocorticoids are as effective as high-dose in treatment of allergic bronchopulmonary aspergillosis http://ow.ly/TLt4N

For editorial comment see Eur Respir J 2016; 47: 385-387 [DOI: 10.1183/13993003.01816-2015].

This article has supplementary material available from erj.ersjournals.com

Received: Sept 042015 | Accepted after revision: Oct 122015 | First published online: Nov 192015

This article has supplementary material available from erj.ersjournals.com

Clinical trial: This study is registered at www.clinicaltrials.gov with identifier number NCT00974766.

Conflict of interest: None declared.

Copyright OERS 2016 


\section{Introduction}

Allergic bronchopulmonary aspergillosis (ABPA) is a complex pulmonary disorder caused by immunologic reactions mounted against antigens of Aspergillus fumigatus [1]. The disease complicates the course of patients with asthma (and cystic fibrosis) [2], clinically manifesting as uncontrolled asthma, recurrent pulmonary opacities and bronchiectasis [3]. The disorder may affect as many as 5 million individuals worldwide [4] and is especially common in India [5, 6]. Despite six decades of research [7], ABPA is still misdiagnosed in as high as one-third of cases [8]. If not adequately treated, the course of the disease is characterised by recurrent episodes of exacerbations, progressing relentlessly to culminate in bronchiectasis and respiratory failure. However, the interest in allergic aspergillosis stems from the fact that the condition is treatment-responsive, and early diagnosis and treatment can prevent progression to end-stage lung disease. The disease course of ABPA can be classified into seven stages [9] and the patient does not necessarily progress from one stage to the other sequentially.

Glucocorticoids are considered the treatment of choice in acute-stage (stage 1) ABPA [10, 11], although there are no placebo-controlled trials of corticosteroids in ABPA. However, their effectiveness in ABPA as observed from years of experience is so well established that it might be considered unethical to conduct placebo-controlled trials $[1,9,12,13]$. Unfortunately, the optimal dose and duration of steroids in the management of ABPA is also not known. Two doses of glucocorticoids have been suggested in acute-stage ABPA. In the medium-dose regime [14], the dose of steroid is prednisone equivalent of $0.5 \mathrm{mg} \cdot \mathrm{kg}^{-1} \cdot \mathrm{day}^{-1}$ for 1-2 weeks, then on alternate days for 6-8 weeks, followed by tapering of 5-10 mg every 2 weeks, until discontinuation [15]. In the high-dose protocol, the dose used is prednisone equivalent of $0.75 \mathrm{mg} \cdot \mathrm{kg}^{-1} \cdot \mathrm{day}^{-1}$ for 6 weeks, then $0.5 \mathrm{mg} \cdot \mathrm{kg}^{-1} \cdot \mathrm{day}^{-1}$ for 6 weeks, followed by tapering of $5 \mathrm{mg}$ every 6 weeks to complete a total duration of 6-12 months [16]. The two protocols have never been compared and the choice between the two remains a matter of personal (or institutional) preference. Nevertheless, there is some suggestion that the use of high-dose steroid, due to its profound anti-inflammatory action early in the course, is associated with a lower rate of progression to glucocorticoid-dependent ABPA [17].

We hypothesised that the high-dose steroid regimen would be superior to the medium-dose protocol in preventing exacerbations and progression to the glucocorticoid-dependent stage in patients with ABPA. In this randomised controlled trial (RCT), we compare the efficacy and safety of the two steroid protocols in the initial management of patients with ABPA complicating asthma.

\section{Material and methods}

This was a prospective, open-label, investigator-initiated RCT conducted in the Chest Clinic of the Postgraduate Institute of Medical Education and Research (PGIMER), Chandigarh, India. The subjects were enrolled between January 2010 and March 2011, and scheduled for follow-up for at least 2 years. The study protocol was approved by the Ethics Review Committee (MS/723/Res/276) and written informed consent was obtained from all subjects. The trial is registered at www.clinicaltrials.gov (NCT00974766).

\section{Patient selection}

Subjects were eligible for inclusion in the study if they were treatment-naive and met the criteria for ABPA defined by the presence of all of the following: 1) asthma, 2) immediate cutaneous hyperreactivity on Aspergillus skin test or A. fumigatus specific IgE levels $>0.35 \mathrm{kUA} \cdot \mathrm{L}^{-1}$, 3) elevated total $\operatorname{IgE}$ levels $>1000 \mathrm{IU} \cdot \mathrm{mL}^{-1}$, and two of the following features: i) presence of precipitating antibodies against A. fumigatus in serum, ii) fixed or transient radiographic pulmonary opacities, iii) total eosinophil count $>1000 \cdot \mu \mathrm{L}^{-1}$ and iv) bronchiectasis on high-resolution computed tomography (HRCT) of the thorax. Subjects with any of the following were excluded: 1) intake of systemic glucocorticoids for $>3$ weeks in the preceding 6 months, 2) immunosuppressive states such as uncontrolled diabetes mellitus, chronic renal failure, chronic liver failure and others, 3) patient on immunosuppressive drugs, 4) pregnancy, 5) enrolment in another trial of ABPA and 6) failure to provide informed consent.

\section{Randomisation}

Subjects meeting the inclusion criteria were randomised 1:1 to either the high-dose or the medium-dose glucocorticoid group (figure 1). The randomisation sequence was computer-generated and the assignments were placed in opaque, sealed envelopes. The study was not blinded.

\section{Study procedure}

After a detailed clinical history and physical examination, all asthmatic subjects were investigated with Aspergillus skin test, IgE levels (total and A. fumigatus-specific), precipitating antibodies against A. fumigatus, peripheral blood eosinophil count, spirometry, chest radiograph and chest HRCT. Chest HRCT was used to classify ABPA as serologic ABPA, ABPA with bronchiectasis or ABPA with high-attenuation mucus [18]. The detailed methodology of each of the aforementioned investigations has been previously described [8, 19-27]. 


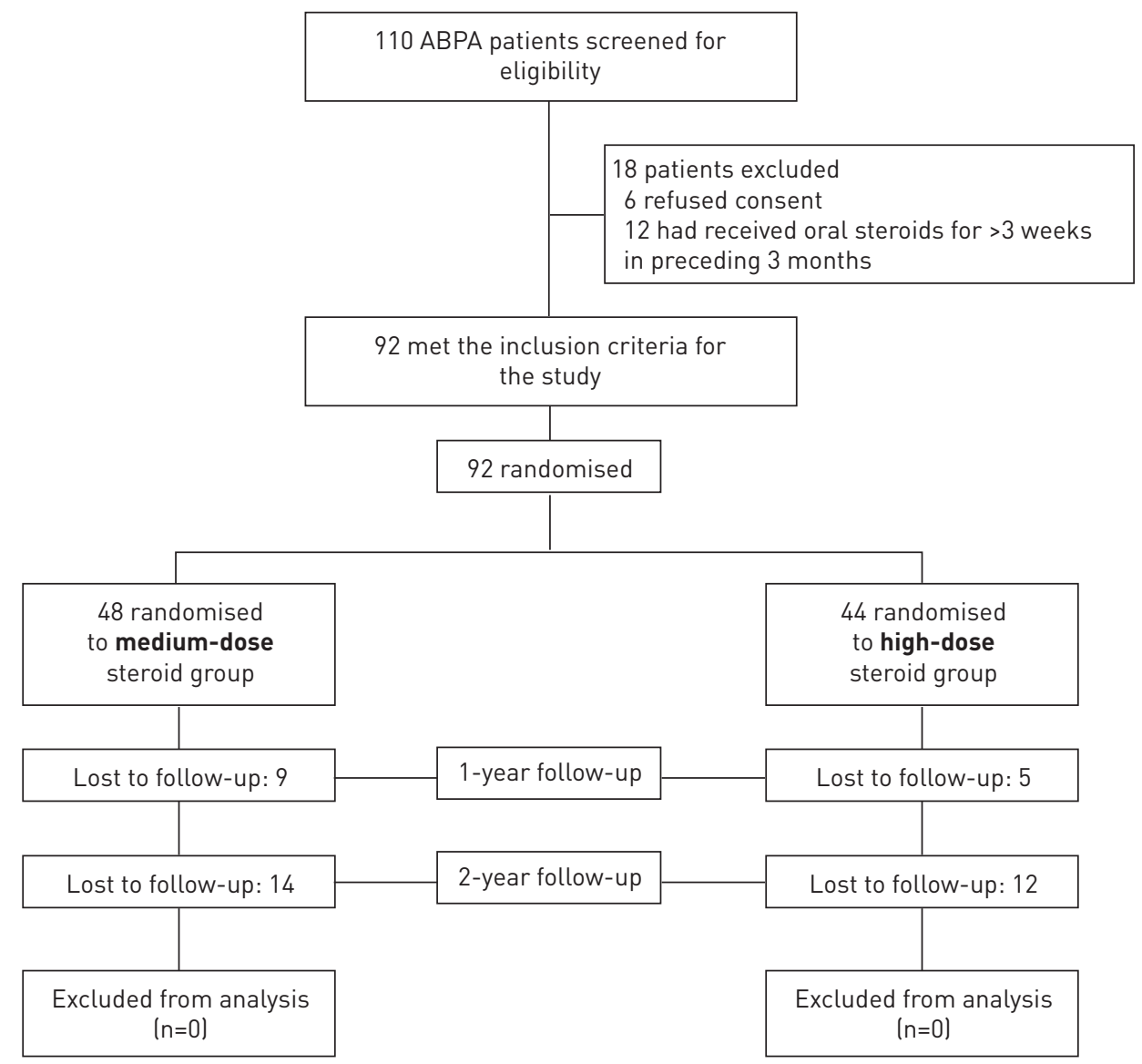

FIGURE 1 CONSORT diagram demonstrating the flow of participants in the study. ABPA: allergic bronchopulmonary aspergillosis.

Subjects fulfilling the inclusion criteria were randomised into one of the following two groups: 1) medium-dose steroids: oral prednisolone $0.5 \mathrm{mg} \cdot \mathrm{kg}^{-1} \cdot \mathrm{day}^{-1}$ for 2 weeks followed by $0.5 \mathrm{mg} \cdot \mathrm{kg}^{-1} \mathrm{on}$ alternate days for 8 weeks, then taper by $5 \mathrm{mg}$ every 2 weeks and discontinue after 3-5 months, and 2) high-dose steroids: oral prednisolone $0.75 \mathrm{mg} \cdot \mathrm{kg}^{-1} \cdot \mathrm{day}^{-1}$ for 6 weeks followed by $0.5 \mathrm{mg} \cdot \mathrm{kg}^{-1} \cdot \mathrm{day}^{-1}$ for 6 weeks, then taper by $5 \mathrm{mg}$ every 6 weeks and discontinue after $8-10$ months. If any subject required the concomitant use of other medications for treatment of ABPA, such as itraconazole, voriconazole or inhaled amphotericin B, for concomitant aspergilloma, then he/she was excluded from the study. The use of inhaled corticosteroids and long-acting $\beta 2$-agonists, and leukotriene receptor antagonists, for the control of asthma was allowed in both the arms, at the discretion of the treating physician.

Apart from the aforementioned investigations, all patients also underwent liver and renal function tests, fasting plasma glucose, and complete blood count. Subjects were followed up with history and physical examination, chest radiograph, and serum IgE levels (total) at each visit every 6 weeks. Spirometry was repeated at the first visit. The patients were clinically monitored for adverse reactions to glucocorticoids such as cataract, glaucoma and others; fasting plasma glucose and blood pressure measurements were performed every 2 months.

\section{End-points}

Clinical improvement in cough and dyspnoea was documented subjectively on a four-point scale as: 1 , no improvement or worsening; 2, mild improvement (up to $25 \%$ of baseline); 3 , moderate improvement (25\%-75\% of baseline); 4 , significant improvement ( $>75 \%$ of baseline). The treatment effects were classified as: 1) composite response (defined after 6 weeks of treatment, based on a combination of clinical, radiological and immunological criteria when there was improvement in cough and dyspnoea ( $>75 \%$ of baseline), and the IgE levels declined by $>25 \%$ accompanied by partial/total clearance of chest radiographic lesions (if present prior to treatment initiation)), 2) exacerbation (clinical and/or radiological worsening along with doubling of the serum total IgE over the previous baseline value) or 3) glucocorticoid-dependent ABPA (if the subject had exacerbation on two or more consecutive occasions 
within 6 months of stopping treatment or required oral steroids for control of asthma). Exacerbations were treated with oral steroids $\left(0.5 \mathrm{mg} \cdot \mathrm{kg}^{-1} \cdot \mathrm{day}^{-1}\right.$ for 4 weeks, then $0.25 \mathrm{mg} \cdot \mathrm{kg}^{-1} \cdot \mathrm{day}^{-1}$ for 4 weeks, then $0.125 \mathrm{mg} \cdot \mathrm{kg}^{-1} \cdot \mathrm{day}^{-1}$ for 4 weeks, then decreased by $5 \mathrm{mg} \cdot \mathrm{week}^{-1}$ for a total of 4 months) and oral itraconazole (200 mg twice daily for 6 months).

The primary outcomes were: 1) exacerbation rates after 12 months of glucocorticoid therapy and 2) progression to glucocorticoid-dependent ABPA after 24 months of treatment. The secondary outcomes were: 1) proportion of subjects with a composite response (as defined above) at 6 weeks of treatment, 2) percentage decline in $\operatorname{IgE}$ (baseline $\operatorname{IgE}$ minus $\operatorname{IgE}$ after 6 weeks/baseline $\operatorname{IgE}$ ) after 6 weeks of treatment, 3) improvement in lung function after 6 weeks of treatment, 4) time to first exacerbation after stopping treatment, 5) cumulative dose of oral glucocorticoids received and 6) glucocorticoid-related adverse effects.

\section{Sample size}

The sample size was calculated based on a reported prevalence of glucocorticoid-dependent ABPA of $45 \%$ in the medium-dose steroid arm compared with $15 \%$ in the high-dose group [16, 28]. With these assumptions, 44 subjects would be required in each arm (confidence level $(1-\alpha) 95 \%$, power level $(1-\beta)$ $80 \%)$ to detect significant differences.

\section{Statistical analysis}

Data were analysed using SPSS (version 22.0; IBM, New York, NY, USA). Statistical significance was assumed at $\mathrm{p}<0.05$. The differences between categorical variables were analysed using the Chi-squared test (or Fisher's exact test); the differences between continuous variables were analysed using the MannWhitney U-test. An intention-to-treat analysis was performed. Survival curves were constructed to study the effect of steroid protocols on the time to first exacerbation using Kaplan-Meier analysis and the group differences were analysed using the log-rank test.

\section{Results}

Of the 110 ABPA subjects screened for eligibility, 18 were excluded before randomisation (figure 1). 92 subjects with ABPA were randomised to either the high-dose $(n=44)$ or the medium-dose steroid group $(\mathrm{n}=48)$. The subject withdrawal rate was similar in the two groups at 1 year (high-dose $5(11.4 \%)$ versus medium-dose $9(18.8 \%)$ ) and 2 years (high-dose 12 (27.3\%) versus medium-dose $14(29.2 \%)$ ). The baseline characteristics were similar in the two groups (table 1). The subjects were generally young with a median (interquartile range (IQR)) age of 31 (22-44) years; 55\% were men. Most subjects had long-standing asthma with a median (IQR) symptom duration of 12 (5-16) years. About $27 \%$ of the subjects had inappropriately received antituberculosis therapy. Approximately $70 \%$ of the study population had abnormal spirometry; percentage of the lower limit of normal forced expiratory volume in $1 \mathrm{~s}$ was $<60 \%$ in $\sim 40 \%$ of the study population. Chest radiograph was abnormal in $62 \%$, while fleeting opacities were observed in $52 \%$ of the subjects. The majority of the subjects (72\%) had bronchiectasis on chest HRCT; high-attenuation mucus was observed in $27 \%$ of the subjects. The immunological investigations were also similar in the two groups (table 1). All the patients were on inhaled corticosteroids and long-acting $\beta 2$-agonist, while $22(23.9 \%)$ patients were also receiving leukotriene receptor antagonists.

\section{Primary outcomes}

The median (IQR) duration of follow-up of the study population was 24 (23-44) months. Overall, 42 (45.7\%) subjects experienced an exacerbation after completion of treatment. The numbers of subjects with exacerbations after 1 year of treatment were similar in the two groups (table 2). The exacerbations were treated with a combination of glucocorticoids and itraconazole. There was good response and only 12 of the 42 subjects were classified as glucocorticoid-dependent ABPA. The numbers of subjects with glucocorticoid-dependent ABPA after 2 years of treatment also did not differ between the two groups (table 2). An exploratory worst-case scenario analysis was also performed assuming all dropout cases to have adverse outcomes. There was no significant change in the relative primary outcomes in the two groups.

\section{Secondary outcomes}

After 6 weeks of treatment, the proportion of subjects with a composite response was higher in the high-dose $(44 / 44,100 \%)$ compared with the medium-dose $(42 / 48 ; 87.5 \%)$ group ( $\mathrm{p}=0.045$; table 2$)$. Similarly, the percentage decline in IgE was also significantly higher in the high-dose group. The improvement in lung function on spirometry at 6 weeks (table 2) and the time to first exacerbation after stopping treatment (figure 2) was similar in the two groups (mean 132 versus 100 days). The total dose (mean 4011 versus $1694 \mathrm{mg}$ ) of corticosteroids was significantly higher in the high-dose arm (table 2). The occurrence of adverse reactions to glucocorticoids such as cushingoid habitus, weight gain and striae 
TABLE 1 Baseline characteristics of the study population

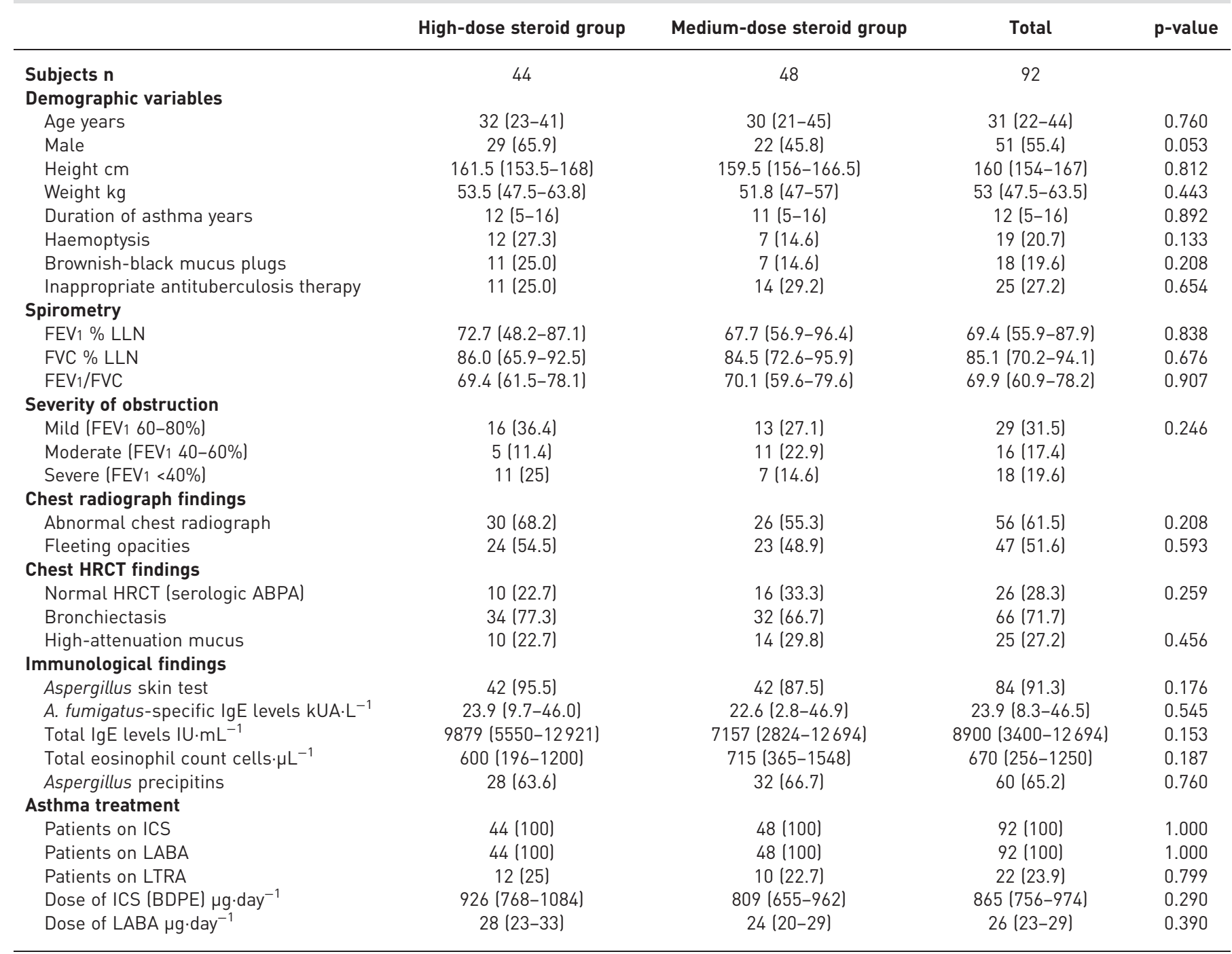

All values are presented as median (interquartile range) or $\mathrm{n}(\%)$, unless otherwise stated. FEV 1 : forced expiratory volume in $1 \mathrm{~s}$; LLN: lower limit of normal; FVC: forced vital capacity; HRCT: high-resolution computed tomography; ABPA: allergic bronchopulmonary aspergillosis; A. fumigatus: Aspergillus fumigatus; ICS: inhaled corticosteroid; LABA: long-acting 32 -agonist; LTRA: leukotriene receptor antagonist; BDPE: beclomethasone equivalent.

formation was also significantly higher in the high-dose arm (table 2). There was no occurrence of pulmonary tuberculosis or nontuberculous mycobacterial infection in the study population.

A subgroup analysis was performed to examine the effect of glucocorticoid dosing on outcomes in patients with high-attenuation mucus (online supplementary table S1). There was no difference in the relative primary or secondary outcome in patients with high-attenuation mucus compared with the overall analysis.

The clinical characteristics of the six subjects who did not respond with medium-dose steroids at 6 weeks are shown in table 3. Although the prevalence of bronchiectasis and the number of segments involved by bronchiectasis was higher in the subjects without response, these differences were not significantly different from the responders (table 4). All the six subjects were treated with high doses of steroids, and showed clinical and/or radiological, and immunological response.

\section{Discussion}

The results of this study suggest that medium-dose oral steroid is as effective as high-dose oral steroid in preventing exacerbations and progression to the glucocorticoid-dependent stage in ABPA complicating asthma. Although composite response rates and percentage decline in IgE were significantly higher in the high-dose arm, improvement in lung function and time to first exacerbation were similar in the two 


\section{Subjects $\mathbf{n}$}

\section{Primary outcomes}

Subjects with exacerbations after 1 year of treatment

Subjects with glucocorticoid-dependent ABPA after 2 years of treatment

\section{Secondary outcomes}

Response after 6 weeks of treatment

Percentage decline in IgE after 6 weeks of treatment

Difference in FEV 1 after 6 weeks of treatment $L$

Difference in FVC after 6 weeks of treatment $L$

Time to first exacerbation after stopping therapy days

Total amount of glucocorticoid mg

Glucocorticoid-related adverse reactions

Cushingoid habitus

Hypertension

Hyperglycaemia

Hypertrichosis

Acne

Striae

Weight gain ( $>10 \%$ of baseline)

Mood changes
44

$$
\begin{gathered}
18(40.9 ; 27.7-55.6) \\
5(11.4 ; 4.9-23.9) \\
\\
44(100 ; 91.9-100) \\
43.8(36.8-50.9) \\
0.27(0.17-0.37) \\
0.37(0.19-0.54) \\
132(84-180) \\
4011(3620-4401) \\
\\
35(79.6 ; 65.5-88.9) \\
1(2.3 ; 0.4-11.8) \\
1(2.3 ; 0.4-11.8) \\
5(11.4 ; 4.9-23.9) \\
16(36.4 ; 23.8-51.1) \\
8(18.2 ; 9.5-31.9) \\
24(54.6 ; 40.1-68.3) \\
4(9.1 ; 3.6-21.2)
\end{gathered}
$$

48

$\begin{array}{cc}24(50 ; 36.4-63.6) & 0.592 \\ 7(14.6 ; 7.3-27.2) & 0.882 \\ & \\ 42(87.5 ; 75.3-94.1) & 0.045 \\ 11.8(-8.1-31.7) & 0.025 \\ 0.34(0.23-0.45) & 0.426 \\ 0.37(0.26-0.49) & 0.725 \\ 100(65-136) & 0.262 \\ 1694(1578-1810) & 0.0001 \\ 14(29.2 ; 18.2-43.2) & 0.0001 \\ 0 & \\ 0 & \\ 0 & 0.098 \\ 10(20.8 ; 11.7-34.3) & 0.025 \\ 1(2.1 ; 0.4-10.9) & 0.0001 \\ 8(16.7 ; 8.7-29.6) & 0.594 \\ 2(4.2 ; 1.2-13.9) & \end{array}$

All data are presented as $\mathrm{n}(\% ; 95 \% \mathrm{Cl})$ or mean $(95 \% \mathrm{CI})$, unless otherwise stated. All outcomes are based on an intention-to-treat analysis. ABPA: allergic bronchopulmonary aspergillosis; FEV1: forced expiratory volume in 1 s; FVC: forced vital capacity.

groups. Total glucocorticoid dose and frequency of adverse events were also significantly higher in the high-dose group. The current study is the largest randomised trial in ABPA and, to the best of our knowledge, the first study evaluating the dose of steroids to be used in the initial management of previously untreated ABPA. Few randomised trials have evaluated the treatment effects in patients with ABPA [29, 30]. The previous two RCTs had investigated the effects of antifungal azoles and were conducted either in steroid-dependent patients or in those with clinically stable disease following steroid therapy [29, 30]. Apart from the small sample size of these RCTs (55 and 29 patients), both the trials followed up patients for a maximum of only 4-8 months.

The principles in the management of ABPA revolve around the use of anti-inflammatory therapy (systemic glucocorticoids) to restrain the immune hyperactivity and the use of antifungal agents (azoles) to mitigate the fungal burden in the airways [31]. Oral corticosteroids are currently the treatment of choice in the management of acute-stage ABPA [32]. ABPA is characterised by increased Aspergillus-induced Th2/ Th1 T-helper cytokine ratio due to activation of inflammatory genes [33]. Glucocorticoids suppress multiple inflammatory genes activated in asthmatic airways by recruiting histone deacetylases and reversing histone acetylation of the activated inflammatory genes [34].

FIGURE 2 Time to first exacerbation in patients receiving high-dose versus medium-dose glucocorticoids. Time to first exacerbation was similar in the two groups.

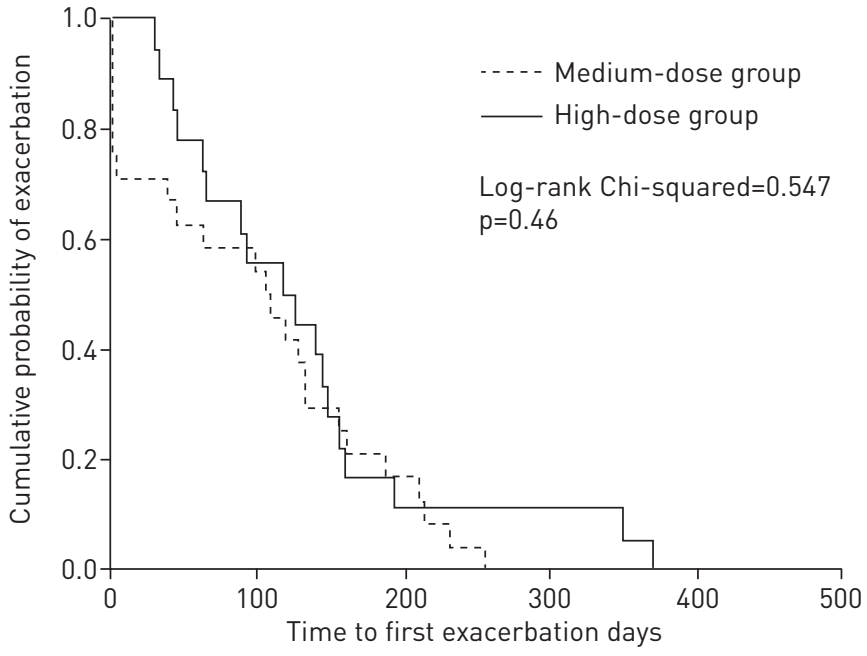


TABLE 3 Clinical characteristics of the six subjects in the medium-dose steroid group with lack of composite response after 6 weeks of treatment

\begin{tabular}{|c|c|c|c|c|c|c|c|c|}
\hline Subject & Age/sex & $\begin{array}{c}\text { Duration of } \\
\text { asthma years }\end{array}$ & $\begin{array}{c}\text { Total IgE } \\
\text { levels IU.mL }\end{array}$ & $\begin{array}{l}\text { A. fumigatus-specific } \\
\text { IgE levels kUA. }{ }^{-1}\end{array}$ & $\begin{array}{l}\text { Total eosinophil } \\
\text { count cells } \mu \mathrm{L}^{-1}\end{array}$ & $\begin{array}{l}\text { HRCT evidence of } \\
\text { bronchiectasis }\end{array}$ & $\begin{array}{c}\text { Segments } \\
\text { involved by } \\
\text { bronchiectasis } n\end{array}$ & $\begin{array}{c}\text { High-attenuation } \\
\text { mucus on chest } \\
\text { HRCT }\end{array}$ \\
\hline 1 & $19 /$ male & 16 & 11058 & 41.1 & 540 & Yes & 8 & No \\
\hline 2 & $32 /$ male & 14 & 1012 & 15.0 & 400 & No & 0 & No \\
\hline 3 & $24 /$ female & 14 & 7105 & 42.9 & 660 & Yes & 5 & No \\
\hline 4 & $20 /$ male & 10 & 11483 & 56.7 & 848 & Yes & 13 & Yes \\
\hline 5 & $20 /$ female & 7 & 19600 & 1.8 & 1840 & Yes & 10 & Yes \\
\hline 6 & $25 /$ female & 1.5 & 12794 & 31.9 & 2440 & Yes & 1 & No \\
\hline
\end{tabular}

HRCT: high-resolution computed tomography; A. fumigatus: Aspergillus fumigatus.

The results of the present study are applicable only to patients with acute-stage ABPA, i.e. stage 1 [9]. In patients with exacerbations, i.e. stage 3 [9], the current treatment of choice is a combination of glucocorticoids and itraconazole $[29,30]$. An interesting proposition is the use of itraconazole monotherapy as first-line therapy for acute-stage ABPA [35], as this would avoid the adverse effects of glucocorticoid therapy altogether. However, any azole therapy has to be balanced in light of the emerging importance of azole-resistant Aspergillus species not only in Europe but also in Asia [36, 37]. Two randomised trials evaluating the role of itraconazole and voriconazole monotherapy in treatment-naive ABPA subjects have been completed (www.clinicaltrials.gov NCT01321827 and NCT01621321), and hopefully the results of these trials should provide clarify the role of azole monotherapy in acute-stage ABPA.

We had hypothesised that treatment of acute-stage ABPA with higher dose and prolonged duration of steroids would be more effective than treatment with lower doses, due to the early and profound suppression of immune hyperfunction. The proportion of patients progressing to corticosteroid-dependent ABPA reported previously was higher in one study using a medium-dose steroid regime $(38 / 84 ; 45.2 \%)$ [28] compared with another study that employed high doses (17/126; 13.4\%) [16]. In the current study, the prevalence of steroid-dependent ABPA was low in both the arms (15\% and $11 \%$ in high-dose and

TABLE 4 Baseline characteristics of the responders and nonresponders in the medium-dose steroid group at 6 weeks

\begin{tabular}{|c|c|c|c|}
\hline & Responders & Nonresponders & p-value \\
\hline Subjects $\mathrm{n}$ & 42 & 6 & \\
\hline \multicolumn{4}{|l|}{ Demographic variables } \\
\hline Age years & $31(22-45)$ & $22(20-25)$ & 0.119 \\
\hline Male & $19(45.2)$ & $3(50)$ & 0.827 \\
\hline Duration of asthma years & $11(5-17.5)$ & $12(7-14)$ & 0.781 \\
\hline \multicolumn{4}{|l|}{ Spirometry } \\
\hline FEV 1 L & $1.99(1.42-2.60)$ & $1.57(1.16-2.08)$ & 0.154 \\
\hline FVC L & $2.86(2.21-3.36)$ & $2.63(1.72-2.81)$ & 0.195 \\
\hline $\mathrm{FEV}_{1} / \mathrm{FVC}$ & $71.5(59.5-79.9)$ & $60.9(59.7-63.4)$ & 0.092 \\
\hline \multicolumn{4}{|l|}{ Chest radiograph findings } \\
\hline Abnormal chest radiograph & $21(51.2)$ & 5 (83.3) & 0.139 \\
\hline Fleeting opacities & 18 (43.9) & 5 (83.3) & 0.071 \\
\hline \multicolumn{4}{|l|}{ Chest HRCT findings } \\
\hline Bronchiectasis & 27 (64.3) & $5(83.3 \%)$ & 0.355 \\
\hline Segments involved & $4(0-8)$ & $7(1-10)$ & 0.363 \\
\hline High-attenuation mucus & $12(29.3)$ & 2 (33.3) & 0.839 \\
\hline \multicolumn{4}{|l|}{ Immunological findings } \\
\hline A. fumigatus-specific IgE levels $\mathrm{kUA} \cdot \mathrm{L}^{-1}$ & $22.6(2.8-46.9)$ & $23.9(9.7-46.0)$ & 0.473 \\
\hline Total IgE levels IU. $\mathrm{mL}^{-1}$ & $5825(2808-12594)$ & $11271(7105-12794)$ & 0.418 \\
\hline Total eosinophil count cells. $\mu \mathrm{L}^{-1}$ & $715(300-1520)$ & $754(540-1840)$ & 0.483 \\
\hline
\end{tabular}

All data are presented as median (interquartile range) or $\mathrm{n}(\%)$, unless otherwise stated. $\mathrm{FEV}$ 1: forced expiratory volume in $1 \mathrm{~s}$; FVC: forced vital capacity; HRCT: high-resolution computed tomography; A. fumigatus: Aspergillus fumigatus. 
medium-dose groups, respectively). This is probably due to improvements in management of asthma over time, with the availability of not only better drugs (including long-acting $\beta 2$-agonists, leukotriene-receptor antagonists and others) [38], but also better approaches [39]. It is also plausible that the eosinophil-predominant inflammation encountered in ABPA is highly responsive to steroids; thus, a lower dose and shorter duration may be as effective as higher doses.

The response rates and percentage decline in $\operatorname{IgE}$ at 6 weeks were significantly higher in the high-dose group. However, this does not support the routine use of high-dose steroids in preference to medium-dose steroids. The majority of the patients in the medium-dose arm showed response at 6 weeks, but high-dose steroids had no impact on the frequency of exacerbations and development of steroid-dependent ABPA. Importantly, improvement in spirometric parameters at 6 weeks and time to first exacerbation were similar in the two groups, which further suggests that the $\operatorname{IgE}$ decline is of little significance. The clinical significance of a decline in serum IgE is not known and in one study the quantum decline in IgE was not a predictor of exacerbation in ABPA [19]. Due to a higher total steroid dose (almost 2.5 times higher), the frequency of adverse events (cushingoid habitus and weight gain) were significantly higher in the high-dose steroid arm. We also did not routinely screen for bone mineral density, adrenal stress response, slit-lamp eye exam and glucose tolerance. The high-dose regime, in particular, is more likely to place subjects at risk for steroid toxicities and is another reason to choose the medium-dose regime given what looks like similar efficacy. Thus, a medium-dose steroid regime is not only equally efficacious as high-dose steroids, but is also less likely to cause adverse effects compared with the high-dose protocol. Therefore, high-dose steroids should not be routinely used in the management of ABPA. However, if the medium-dose protocol is chosen, a failure to respond at 6 weeks should promptly trigger consideration for higher doses of steroids. Unfortunately, the study could not identify any factor that could predict failure of response to medium-dose steroids.

Finally, the study is not without limitations. This was a single-centre study and due to logistic difficulties, the study could not be blinded. To overcome this limitation, the clinical and radiological improvement was corroborated by an objective marker, i.e. IgE levels. The study also did not utilise any quality of life scores, although patient-centric outcomes such as clinical improvement were scored on a semi-quantitative Likert scale. Furthermore, we measured spirometry at baseline and at 6 weeks to complement the clinical, radiological and immunological improvement. The study enrolled patients with ABPA complicating asthma only, thus the results may not be applicable to other patient populations (such as cystic fibrosis) with ABPA. There was considerable attrition of study participants at 1 and 2 years of follow-up; however, the dropout rate was similar in the two groups. The loss to follow-up does not appear to be linked to dose-related adverse events as the high-dose regime had fewer dropouts. It is likely that improvement in the underlying condition prompted them to stop further follow-up. However, a worst-case scenario analysis was also performed with the missing data [40] and there was no change in the study outcomes. In any event, the balance of safety and effectiveness clearly supports the low dosage over the higher dosage treatment for most subjects. Although the sample size was calculated a priori, the presumed difference between the two study arms of $30 \%$ was not seen and thus the study may be underpowered to detect the differences. In fact, with the current difference of $4 \%$ in the two arms, a sample of 1159 subjects in each arm would be required to detect any significant difference. If we use figures from the current study for calculating sample size for the exacerbation rate, 502 patients will be required in each arm to detect differences. Therefore, the results need to be verified in a larger, preferably multicenter trial. Finally, the trial was conducted at a tertiary-care referral centre and thus there may be limitations in terms of applicability to other settings.

In conclusion, medium-dose oral steroids may be as effective and safer compared with high-dose steroids in preventing exacerbations and glucocorticoid dependence in ABPA. However, a small proportion of patients do not have an adequate early response to medium-dose steroids; hence, the initial treatment needs to be closely monitored.

\section{References}

$1 \quad$ Agarwal R. Allergic bronchopulmonary aspergillosis. Chest 2009; 135: 805-826.

2 Chung KF, Wenzel SE, Brozek JL, et al. International ERS/ATS guidelines on definition, evaluation and treatment of severe asthma. Eur Respir J 2014; 43: 343-373.

3 Kosmidis C, Denning DW. The clinical spectrum of pulmonary aspergillosis. Thorax 2015; 70: 270-277.

4 Denning DW, Pleuvry A, Cole DC. Global burden of allergic bronchopulmonary aspergillosis with asthma and its complication chronic pulmonary aspergillosis in adults. Med Mycol 2013; 51: 361-370.

5 Agarwal R, Denning DW, Chakrabarti A. Estimation of the burden of chronic and allergic pulmonary aspergillosis in India. PLoS One 2014; 9: e114745.

6 Agarwal R. Burden and distinctive character of allergic bronchopulmonary aspergillosis in India. Mycopathologia 2014; 178: 447-456.

7 Hinson KF, Moon AJ, Plummer NS. Broncho-pulmonary aspergillosis; a review and a report of eight new cases. Thorax 1952; 7: 317-333. 
Agarwal R, Gupta D, Aggarwal AN, et al. Clinical significance of hyperattenuating mucoid impaction in allergic bronchopulmonary aspergillosis: an analysis of 155 patients. Chest 2007; 132: 1183-1190.

9 Agarwal R, Chakrabarti A, Shah A, et al. Allergic bronchopulmonary aspergillosis: review of literature and proposal of new diagnostic and classification criteria. Clin Exp Allergy 2013; 43: 850-873.

10 Patterson KC, Strek ME. Diagnosis and treatment of pulmonary aspergillosis syndromes. Chest 2014; 146: $1358-1368$.

11 Moss RB. Treatment options in severe fungal asthma and allergic bronchopulmonary aspergillosis. Eur Respir J 2014; 43: 1487-1500.

12 Greenberger PA. Diagnosis and management of allergic bronchopulmonary aspergillosis. Allergy Proc 1994; 15: $335-339$.

13 Greenberger PA. Allergic bronchopulmonary aspergillosis. In: Grammer LC, Greenberger PA, eds. Patterson's allergic diseases. Philadelphia, Lippincott, Williams \& Wilkins, 2002; pp. 529-554.

14 Buttgereit F, da Silva JA, Boers M, et al. Standardised nomenclature for glucocorticoid dosages and glucocorticoid treatment regimens: current questions and tentative answers in rheumatology. Ann Rheum Dis 2002; 61: 718-722.

15 Greenberger PA. Allergic bronchopulmonary aspergillosis. J Allergy Clin Immunol 2002; 110: 685-692.

16 Agarwal R, Gupta D, Aggarwal AN, et al. Allergic bronchopulmonary aspergillosis: lessons from 126 patients attending a chest clinic in north India. Chest 2006; 130: 442-448.

17 Bains SN, Judson MA. Allergic bronchopulmonary aspergillosis. Clin Chest Med 2012; 33: $265-281$.

18 Agarwal R, Khan A, Garg M, et al. Pictorial essay: allergic bronchopulmonary aspergillosis. Indian J Radiol Imaging 2011; 21: 242-252.

19 Agarwal R, Gupta D, Aggarwal AN, et al. Clinical significance of decline in serum IgE levels in allergic bronchopulmonary aspergillosis. Respir Med 2010; 104: 204-210.

20 Agarwal R, Nath A, Aggarwal AN, et al. Aspergillus hypersensitivity and allergic bronchopulmonary aspergillosis in patients with acute severe asthma in a respiratory intensive care unit in North India. Mycoses 2010; 53: $138-143$.

21 Agarwal R, Noel V, Aggarwal AN, et al. Clinical significance of Aspergillus sensitisation in bronchial asthma. Mycoses 2011; 54: e531-e538.

22 Agarwal R, Aggarwal AN, Garg M, et al. Allergic bronchopulmonary aspergillosis with aspergilloma: an immunologically severe disease with poor outcome. Mycopathologia 2012; 174: 193-201.

23 Agarwal R, Garg M, Aggarwal AN, et al. Serologic allergic bronchopulmonary aspergillosis (ABPA-S): long-term outcomes. Respir Med 2012; 106: 942-947.

24 Agarwal R, Khan A, Garg M, et al. Chest radiographic and computed tomographic manifestations in allergic bronchopulmonary aspergillosis. World J Radiol 2012; 4: 141-150.

25 Agarwal R, Maskey D, Aggarwal AN, et al. Diagnostic performance of various tests and criteria employed in allergic bronchopulmonary aspergillosis: a latent class analysis. PLoS One 2013; 8: e61105.

26 Agarwal R, Aggarwal AN, Garg M, et al. Cut-off values of serum IgE (total and A. fumigatus-specific) and eosinophil count in differentiating allergic bronchopulmonary aspergillosis from asthma. Mycoses 2014; 57: 659-663. Agarwal R, Devi D, Gupta D, et al. A questionnaire-based study on the role of environmental factors in allergic bronchopulmonary aspergillosis. Lung India 2014; 31: 232-236.

28 Patterson R, Greenberger PA, Halwig JM, et al. Allergic bronchopulmonary aspergillosis. Natural history and classification of early disease by serologic and roentgenographic studies. Arch Intern Med 1986; 146: 916-918.

29 Stevens DA, Schwartz HJ, Lee JY, et al. A randomized trial of itraconazole in allergic bronchopulmonary aspergillosis. N Engl J Med 2000; 342: 756-762.

30 Wark PA, Hensley MJ, Saltos N, et al. Anti-inflammatory effect of itraconazole in stable allergic bronchopulmonary aspergillosis: a randomized controlled trial. J Allergy Clin Immunol 2003; 111: 952-957.

31 Moss RB. Critique of trials in allergic bronchopulmonary aspergillosis and fungal allergy. Med Mycol 2006; 44: Suppl. 1, 269-272.

32 Agarwal R, Chakrabarti A. Allergic bronchopulmonary aspergillosis in asthma: epidemiological, clinical and therapeutic issues. Future Microbiol 2013; 8: 1463-1474.

33 Becker KL, Gresnigt MS, Smeekens SP, et al. Pattern recognition pathways leading to a Th2 cytokine bias in allergic bronchopulmonary aspergillosis patients. Clin Exp Allergy 2015; 45: 423-437.

34 Barnes PJ, Adcock IM. How do corticosteroids work in asthma? Ann Intern Med 2003; 139: 359-370.

35 Nepomuceno IB, Esrig S, Moss RB. Allergic bronchopulmonary aspergillosis in cystic fibrosis: role of atopy and response to itraconazole. Chest 1999; 115: 364-370.

36 Verweij PE, Ananda-Rajah M, Andes D, et al. International expert opinion on the management of infection caused by azole-resistant Aspergillus fumigatus. Drug Resist Updat 2015; 21-22: 30-40.

37 Chowdhary A, Sharma C, Kathuria S, et al. Prevalence and mechanism of triazole resistance in Aspergillus fumigatus in a referral chest hospital in Delhi, India and an update of the situation in Asia. Front Microbiol 2015; 6: 428.

38 Global Initiative for Asthma (GINA). Global strategy for asthma management and prevention (2014). www.ginasthma.org/local/uploads/files/GINA_Report_2014_Aug12.pdf Date last accessed: February 17, 2015.

39 Agarwal R, Khan A, Aggarwal AN, et al. Is the SMART approach better than other treatment approaches for prevention of asthma exacerbations? A meta-analysis. Monaldi Arch Chest Dis 2009; 71: 161-169.

Dettori JR. Loss to follow-up. Evid Based Spine Care J 2011; 2: 7-10. 